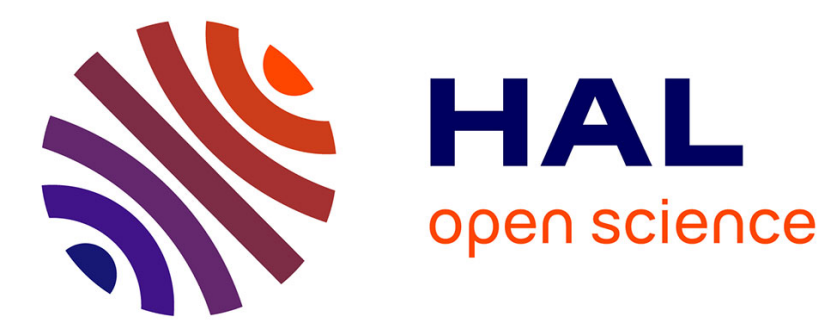

\title{
Psychiser le maître absolu : solutions pubertaires par le paranormal
}

\author{
Renaud Evrard
}

\section{To cite this version:}

Renaud Evrard. Psychiser le maître absolu : solutions pubertaires par le paranormal. Adolescence, 2010, Construction, 74 (4), pp.841-853. 10.3917/ado.074.0841 . halshs-02137282

\section{HAL Id: halshs-02137282 \\ https://shs.hal.science/halshs-02137282}

Submitted on 22 May 2019

HAL is a multi-disciplinary open access archive for the deposit and dissemination of scientific research documents, whether they are published or not. The documents may come from teaching and research institutions in France or abroad, or from public or private research centers.
L'archive ouverte pluridisciplinaire HAL, est destinée au dépôt et à la diffusion de documents scientifiques de niveau recherche, publiés ou non, émanant des établissements d'enseignement et de recherche français ou étrangers, des laboratoires publics ou privés. 


\section{Psychiser le maître absolu : solutions pubertaires par le paranormal}

Renaud Evrard

$\underline{\text { Mesures du paranormal chez les adolescents }}$

L'intérêt d'une majorité d'adolescents pour le paranormal, comme théorie et comme expérience, se lit dans les statistiques de divers sondages, au point de l'élever au rang d'une banalité. C'est surtout parmi les jeunes, notamment ceux qui font des études, que les croyances au paranormal sont les plus fréquentes. Un jeune sur cinq croit aux esprits, un sur trois aux rêves qui prédisent l'avenir, un sur deux à la télépathie (Delestre, 1997). Malheureusement, la plupart des chiffres ou des statistiques sont avancés pour des tranches d'âge plus élevées (18-24 ans).

Selon une étude plus précise réalisée en Alsace sur 384 élèves de collège et lycée (Staehler, 2008), $83 \%$ espèrent qu'il existe quelque chose après la mort, $41 \%$ croient au diable. On savait que les adolescents constituent la classe d'âge la plus croyante aux extraterrestres (Renard, 1988), et cela s'est confirmé dans cette enquête, avec en plus une différence selon les sexes. Ce sont les garçons qui croient le plus au parascientifique des extraterrestres et des soucoupes volantes ; tandis que les filles se tournent vers les croyances plus ésotériques, comme l'astrologie ou la voyance.

Au niveau des mises en pratique de ces croyances, deux adolescents sur trois ont pris part à une séance de spiritisme (Coulombe, 1993). Le nombre de sites internet sur le paranormal qui sont animés par des adolescents montrent comment ce média facilite l'échange autour de ce sujet.

Il faut toutefois distinguer ces réponses à des sondages et les expériences vécues par certains adolescents comme paranormales. Sur ce point, les chiffres manquent. Je parlerais donc à partir de mon expérience clinique. J'ai été engagé par une fondation reconnue d'utilité publique qui cherchait des psychologues pour répondre aux nombreuses demandes de personnes vivant des expériences perçues par elles comme paranormales. Je présenterais deux cas contribuant à ces «cliniques 
adolescentes incluant un élément ou un scénario paranormal, sans qu'on puisse tout de suite évoquer la psychose $»^{1}$.

\section{$\underline{\text { Vignette clinique }}$}

Dans le cas de Julie, 15 ans, je suis d'abord contacté par sa mère. Sa fille s'est rendue dans une maison « hantée » et serait maintenant poursuivie par une entité. Que faire ?

La «maison hantée » est en fait une maison abandonnée dont Julie et deux amis ont forcé la porte. Ils ont joué à se faire peur en visitant les pièces, jusqu'à ce qu'ils entendent un bruit, qu'ils se sentent « poussés par une force » dans les escaliers, et qu'ils ressortent. Julie a alors vu une petite fille à une fenêtre du premier étage, croyant halluciner.

Puis, sur le chemin du collège, elle voit une petite fille derrière un arbre qui l'appelle «dans sa tête », mais elle fait le tour de l'arbre et ne trouve personne. Le petit groupe décide de retourner à la maison en question et là, Julie dit revoir la petite fille et entend dans sa tête comme un appel. Puis c'est le trou noir. Ses amis lui racontent qu'elle serait devenue enragée et qu'elle a essayée de casser la porte, disant qu'il fallait apporter de l'aide.

Depuis, Julie voit cette fille partout et ressent sa souffrance. Elle sent continuellement une présence froide et dit avoir peur. Elle me confie que cela la perturbe au point de la distraire complètement durant les examens scolaires.

En même temps qu'elle dit cela, j'entends qu'elle ne semble pas si préoccupée. J'ai l'impression que cela l'amuse, qu'elle ne ressent pas vraiment le besoin d'y mettre fin. Son interprétation de son expérience n'est pas certaine. Elle dit qu'elle n'y croyait pas trop avant, que « la médiumnité, c'est des bobards », que le problème ne vient pas d'elle mais de cette petite fille probablement entourée d'esprits malins. Julie sépare clairement cette petite fille d'elle-même, ne la percevant pas comme une figure du double. Et quand je tente d'introduire un rapprochement, une spécularité, j'obtiens du 
silence, comme s'il y avait une carence dans les processus de réflexivité.

Cette histoire prendra une autre dimension quand elle fut replacée dans son contexte familial. Il y a huit enfants dans la famille et la plupart des filles ont vécu le même type d'expériences. La mère elle-même croît aux fantômes et pense qu'un de ses ex-maris intervient comme esprit protecteur depuis 20 ans. La mère et plusieurs de ses sœurs aînées auraient même vécu des phénomènes étranges en réaction à l'expérience de Julie.

C'est dire comment cette expérience paranormale était structurante dans ce contexte familial et culturel précis. La mère avait prise Julie entre quatre yeux pour lui narrer cette tradition familiale remontant aux grands-parents, en lui passant notamment des cassettes de la série « Médium » ayant valeur de vérité. C'était une sorte de rite de passage, parce qu'il fallait avoir atteint un certain âge pour entrer dans le secret.

Dans le cas de Julie, ce n'était pas un hasard si le contact vint de la mère, dont la démarche se faisait d'abord à l'insu de sa fille. Il y avait la demande manifeste («Je ne veux pas que les esprits s'en prennent à Julie ») sur laquelle la mère a ensuite associé, m'expliquant se sentir dépassé «par les esprits » avec lesquels elle doit se battre, mais aussi avec Julie qui est une adolescente qui s'occupe plus de son petit ami que de ses études. Cela amène à entendre les symptômes scolaires de Julie différemment, l'alibi d'une gêne occasionnée par le fantôme signifiant un compromis à l'endroit du désir de la mère. En effet, si la mère disait craindre l'influence des esprits sur Julie, que la fille fantôme puisse lui piquer son énergie, elle pensait aussi pouvoir « communiquer très fort » avec ses enfants, prétendant par exemple pouvoir les inciter à rentrer à la maison par des ordres délivrés mentalement. Les craintes maternelles sur «l'influence des esprits » reflètent aussi une crainte d'une perte de contrôle sur cette nouvelle adolescente. Et l'expérience de Julie peut s'entendre comme compromis et échappatoire : avec une autre fille ayant besoin d'aide, elle pourrait peut-être commencer à vivre sa vie comme ses grandes sœurs.

C'est pourquoi les entretiens ont pris fin rapidement avec Julie puisque, selon sa mère, « elle préfère 
garder ses capacités et qu'on traite seulement le problème de cette petite fille ».

\section{$\underline{\text { Interprétations psychosociologiques }}$}

Dans la littérature, cet intérêt des adolescents pour le paranormal a reçu de nombreuses interprétations. Certaines sont très alarmistes et nous parlent d'un retour en force de l'irrationnel, de dérives sectaires, de transgressions sociales par des spiritualités sauvages et archaïques, ou encore d'un business de l'ésotérisme en plein boum. Ces discours proviennent de toutes parts, et même parfois de psychiatres et psychologues (Klosinski, 1997).

Or, pour reprendre la distinction de Lesourd ${ }^{2}$ au sujet de la passion mystique de l'adolescent, il faut différencier la foi, comme passion dévorante du tout, de l'unité, et la croyance marquée de la castration, de la limite. Autrement dit, aucune formation imaginaire n'est spécifique, comme le constatait Lacan dans les années 1950, ce qui a pour conséquence qu'aucune idée n'est délirante en soi, mais qu'il y a uniquement des sujets délirants. Si bien qu'aucune idée étrange investie passionnément par l'adolescent ne saurait-être en elle-même un indice suffisant de ses mouvements psychiques. Il est nécessaire d'entendre par quelles modalités l'adolescent appréhende le paranormal, indépendamment de la forme contingente de son contenu.

Or, la situation est souvent dramatisée par un rapprochement avec la psychopathologie : un suicide d'adolescent sur trois s'inscrirait dans un contexte de cultes sataniques ou de pratiques occultes. C'est ce qui a pu être entendu en mai 2009 sur M6 dans une interview d'un collaborateur de la Miviludes $^{3}$. Aucune étude scientifique ne vient appuyer cette statistique alarmante. Ce discours normatif tombe souvent dans le piège de s'opposer aux ressources culturelles ou contre-culturelles de l'adolescent, en mettant en garde contre les jeux de rôle, l'univers héroïc-fantasy ou même Harry Potter, l'adolescent sorcier dans son école de magie. Notre propos consistera plutôt à sensibiliser à

$2 \quad$ Lesourd, 2008, p. 14.

3 MIVILUDES : Mission Interministérielle de Vigilance et de Lutte contre les Dérives Sectaires. 
la diversité des usages des pratiques occultes au cours du passage adolescent.

Des études par questionnaire pointent la difficulté à faire passer ces scores de croyances au paranormal à une réalité clinique qui met en jeu «l'expérience plus que la croyance » ${ }^{4}$. Ainsi, Hergovich et al. (2008) confirment sur une population d'adolescents de 12 à 19 ans le lien établi entre croyances au paranormal et schizotypie, une forme de schizophrénie latente. Mais ils en viennent à s'interroger sur la pertinence de leurs questionnaires dont les items testent la «pensée magique », capable de décrire à la fois des traits schizophréniques et des perceptions du paranormal. Doit-on attribuer ce chevauchement à celui des catégories qui nous servent à décrire les expériences paranormales ? Les mêmes auteurs identifient la schizotypie adolescente au risque de psychose sans prendre en compte ni les formes non pathologiques de la schizotypie (Goulding, 2005 ; Schofield \& Claridge, 2007) ni la place de la croyance au moment pubertaire (Gutton, 1996), ni la spécificité du fonctionnement adolescent.

\section{Clinique allemande}

Quand on examine cette littérature, on se rend compte que les adolescents ne sont jamais invités à donner les motifs personnels de leur intérêt. Il y a néanmoins des exceptions en Allemagne suite aux travaux du professeur de psychologie Johannes Mischo qui a mené des entretiens approfondis avec plusieurs adolescents en prise avec le paranormal (Mischo, 1991). Selon lui, dans $80 \%$ des cas, le contact avec le paranormal est motivé par la curiosité et l'ennui. Il repère cet intérêt comme un phénomène transitoire, d'une durée comprise entre six semaines et sept mois. Une seule expérience de la richesse imaginaire et émotionnelle du spiritisme ou de l'expérience de hors corps (Le Maléfan, 2005) suffirait à la grande majorité des adolescents. Ce serait alors une forme banale de rencontre avec l'insolite, à rapprocher des rites de passage collectifs ou des auto-initiations.

Cependant, il reste encore $20 \%$ d'adolescents chez qui le contact avec le paranormal s'inscrit dans 
des problématiques plus «profondes » comme le deuil. C'est pourquoi Mischo, en association avec l'Université de Freiburg, a mis en place une clinique pour les personnes vivant des expériences paranormales. Après 20 ans d'activité, les échos de cette clinique permettent de dire que les pratiques occultes restent le premier motif de consultation des adolescents (Lucadou \& Zahradnik, 2001). En moyenne, il s'agit d'adolescents de 16 ans, intelligents et avec de bonnes capacités de verbalisation, qui entreprennent une démarche pour comprendre leur expérience et l'expliquer scientifiquement. Derrière cet abord apparemment rationnel, l'occultisme n'est pas juste un jeu ou une provocation. C'est un lieu pour de nombreux questionnements existentiels sur la vie, la mort et le spirituel (Lucadou, 1992a, 1992b). Les adolescents ont peu d'occasions de discuter de la vision du monde qu'ils se fabriquent et qui les conduit à s'interroger sur ce qu'ils sont, sur l'inscription de leur destin dans leur histoire. C'est pourquoi un tel espace est propice à des prises de parole originales pouvant conduire à des remaniements psychiques.

La diversité des solutions par le paranormal

Même si le paranormal peut soutenir un fonction psychologique, les pratiques de divination ou de séances spirites ne constituent pas en elles-mêmes des solutions viables. Jean-Claude Maleval fait remarquer que la confrontation à l'incomplétude de l'Autre est un des facteurs majeurs de déclenchement de la psychose. «Ainsi interroger l'Autre du savoir absolu, par l'entremise de pratiques de divination ou de séances spirites, peut présenter de réels dangers pour un sujet de structure psychotique, en particulier quand des réponses ambiguës suggèrent l'inhérence au symbolique d'un impossible à dire. ${ }^{5}$ Ces dangers dépendent donc de la structure, et demandent de réfléchir à la façon dont l'occultisme agirait comme revoilement structurant.

Pour faire face aux énigmes concernant le secret du sexuel, l'adolescent peut s'investir dans l'étude de forces cachées qui gouvernent le monde de manière non immédiatement perceptive, ce qui peut le conduire dans des zones présumées « au-delà des apparences » ${ }^{6}$. Le Maléfan (2008) a décrit comment cet intérêt pour le paranormal, ou la parapsychologie,

$5 \quad$ Maleval, 2000, p. 210.

$6 \quad$ Roussillon et al., 2007, p. 205. 
qui est sa version supposée scientifique et rationnelle, pourrait faire avoir un effet structurant face à cette découverte fondamentale de l'incomplétude du symbolique. Ce serait ainsi « un mode à la fois exploratoire des limites du symbolique et des effets du signifiant, et une tentative de baliser ce qui est une des conséquences de la découverte de cette incomplétude, la part de jouissance non phallique à laquelle le sujet se trouve nouvellement confronté » ${ }^{7}$. De sorte que cet intérêt croyant peut venir pour certains sujets prolonger les refuges imaginaires du temps de l'infantile, telle la croyance au Père-Noël ou en des mondes parallèles fantastiques. Comme il serait trop triste de ne pas croire au phallus et à la possibilité de la satisfaction complète, l'adolescent vient mettre en place une certaine croyance qui ne serait pas dans le registre de la foi passionnelle. Il s'agit d'y croire sans y croire, ce qui reflète les formes modernes du croire adolescent: bricolage de croyances flottantes et possibilistes, rapportées à soi, sortant de la religion instituée et même en défiance par rapport aux institutions $^{8}$. Toutefois, dans la majorité des cas, ces croyances se résorbent à l'âge adulte dans un mouvement refoulant qui les situent clairement dans une irréalité. Il s'agirait d'une croyance ayant pour fonction d'être dépassée et invalidée mais qui ne relèverait pas de la psychopathologie ou du mysticisme pour ceux qui la produisent.

On peut comprendre diverses croyances ou pratiques occultes comme des tentatives de revoilement de cette destitution imaginaire de la puissance phallique. L'Autre de l'enfance se révèle en effet ne plus pouvoir incarner la toute-puissance qui lui était prêtée, ce qui remet en question ce qui soutenait la portée de la métaphore paternelle qui s'appuyait imaginairement sur les personnages familiaux. A cause de ce manque dans le registre imaginaire, le sujet est conduit à s'inventer de nouvelles identifications articulées au social. Il doit aussi recomposer avec un fantasme d'immortalité (Gutton, 1993) qui vacille. Cela peut se faire par une preuve par le corps, en survivant à un traumatisme - au moyen parfois d'une conduite ordalique - mais aussi, et peut-être d'une manière plus métaphorique mais non moins agissante - par la prise de contact avec des défunts. Les jeux d'asphyxie se prêtent ainsi à une recherche de sensations de jouissance de la mort imminente, via une entrevue initiatique avec un au-delà, supposée causée par l'anoxie cérébrale (Durastante, 2009 ; Le Maléfan, 2001). L’expression « psychiser le Maître absolu » englobe ces processus par lesquels une élaboration subjective de la mort est faite au moyen de pratiques occultes ordaliques. La pratique des jeux spirites, derrière les motifs conscients de curiosité et d'ennui relevés par Mischo,

$7 \quad$ Le Maléfan, 2008, p. 709.

8 Le Vallois, Aulenbacher, 2006, p. 62-65. 
peut impliquer un essai de mise en rapport avec le réel de la mort.

Le recours au paranormal est l'une des voies transitoires, sous forme de mythologie signifiante, que peut prendre alors le sujet dans une quête d'une consistance nouvelle de l'Autre. L'adolescent fera face à un champ laissé libre à d'autres formules possibles de l'Altérité, dont certaines sont portées par la culture. La première solution envisagée, presque la plus classique, serait de mettre « Dieu » à cette place transcendante. C'est une solution qui semble tout à fait permettre un travail psychique, axé notamment sur les questions de transmission (Falque, 1998 ; Nicabou, 2007). Néanmoins, le désavantage de cette solution est d'appartenir encore trop à l'univers des adultes. Si on doute de ses parents, pourquoi ne douterait-on pas aussi de Lui ? Non seulement l'occultisme et ses « croyances parallèles » pourraient alors se situer comme des alternatives intéressantes mais, d'après les sondages sur ces croyances, elles seraient manifestement déjà largement utilisées.

\section{$\underline{\text { Vignette clinique }}$}

Yann a 15 ans quand je le reçois. A 11 ans, son père a quitté le foyer. Yann associe à ce départ une blessure à la tempe reçue lors d'une bagarre avec des copains « qui le ridiculisent », ditil. Dans un état de grande fragilité psychologique, Yann fait plusieurs tentatives de suicide et se retrouve hospitalisé. Au moment où je le rencontre, il est encore suivi par une psychologue disqualifiée parce qu'elle «ne connaît rien au paranormal ». Or, c'est bien par le paranormal que Yann trouve quelque chose à élaborer. Il a lu Le livre des médiums d'Allan Kardec (1861), le codificateur du spiritisme, et se plaît à croire qu'il est lui-même médium. Plusieurs expériences l'y inclinent : il a fait deux «voyages astraux » (l'appellation ésotérique des expériences de hors corps, cf. Le Maléfan, 2005) en «état d'absence », dont l'un a coïncidé avec une apparition ressentie par une amie vers laquelle il s'était déplacé en imagination. Comme il prenait ses rêves en notes, il y détectait plusieurs éléments qui lui semblaient prémonitoires. Pendant un mois, avec sa mère, ils auraient observé des phénomènes de hantise : bruits inexpliqués, objets mystérieusement déplacés, apparition d'«insectes des marais » en plein salon. Malgré ses difficultés scolaires persistantes, il voulait connaître les « débouchés » de la parapsychologie. 
Quelque chose d'une ambition créative trouvait à s'alimenter par ces vécus. Elle se confrontait néanmoins aux moqueries de ses camarades, devant qui il tentait de reproduire les phénomènes sur demande afin de les convaincre. Depuis qu'il a 14 ans, il assume ces phénomènes en même temps que «sa bizarrerie » qui lui donne de l'importance. Le paranormal vient revoiler une faille narcissique, jouant sur la promesse d'un «corps plus calé » (Lacan, 1975). Ainsi, si la mère autorise l'hypothèse de la médiumnité de son fils, Yann est en conflit ouvert avec son père qui n'entend rien à ses phénomènes, bien que ce dernier pense par ailleurs que les OVNI sont de véritables engins extraterrestres. Yann a trouvé refuge dans un autre appareillage imaginaire : il met directement en lien les phénomènes avec une expression de ses troubles psychologiques, favorisant l'hypothèse «parapsychanalytique » (Calvesi, 1983) d'un adolescent perturbé à l'intérieur qui va déclencher une hantise dans son environnement. Par ce moyen, il peut se réapproprier également son parcours psychiatrique pour en faire quelque chose de plus positif, bien que marginal. Dans cette optique, l'exploration des dispositifs culturels investis par cet adolescent permet de situer ce qu'il vient en retirer, les éléments du discours sur lesquels il se calque, et ceux qui lui sont propres (Evrard, 2008).

Le cas de Yann semble montrer qu'une manière originale d'affronter le manque dans l'ordre symbolique est de se prendre soi-même pour une figure de l'Autre, dans une adhésion à ces fragments de divin que seraient ces pouvoirs. Il ne s'agit pas simplement de croire ou d'interpréter certaines expériences comme paranormales, mais d'être convaincu de posséder de tels pouvoirs de façon permanente, dans un sentiment de puissance et d'exceptionnalité.

\section{$\underline{\text { Conclusion }}$}

Au final, il reste difficile de dire quand le paranormal constitue une solution pour un adolescent. Le même jeu spirite n'aurait pas les mêmes implications suivant la façon dont il s'encastre dans une structure psychotique ou vient revoiler l'opération subjectivante du névrosé. Il semble néanmoins essentiel de démêler, en deux temps, le rapport de l'adolescence et de la psychose et celui de la 
paranormalité et de la psychose. Le Maléfan ${ }^{9}$ préconise l'analyse de davantage de cliniques adolescentes incluant un élément ou un scénario paranormal, sans qu'on puisse tout de suite évoquer la psychose.

Pour expliquer les expériences vécues par ces adolescents, il faudrait aussi porter attention aux concepts d'hallucinations et de délires non pyschotiques (Askenazy et al., 2009 ; Larøi et al., 2009 ; Symann \& Hayez, 2008) que les classifications psychiatriques n'intègrent pas. Le fait de ne plus situer hallucinations et délires uniquement comme symptômes de la psychose invite à repenser à des alternatives. Par exemple, un meilleur pronostic est attribué aux expériences hallucinatoires associées à un vécu d'anxiété et d'étrangeté qu'aux mêmes expériences lorsqu'elles sont moins intériorisées et accompagnées de comportements violents ${ }^{10}$.

De son côté, la clinique structurale n'oppose pas ce qui est bénin et ce qui est grave, mais névrose et psychose comme différents appareillages et équilibrages imaginaires avec lesquels un sujet peut éviter de parler en son nom ${ }^{11}$. La présence du paranormal dans les cliniques adolescentes ne pourraient être que l'effet de la conjonction entre des mouvements psychiques et des références sociales. «En faisant de l'Homme la référence, le nouveau lien social a libéré l'humain de la culpabilité qui était le limon de toutes les religions, et il a mis en avant l'autre figure idéale de l'homme, celle dans laquelle il se prend lui-même pour un dieu, le moi-idéal issu de la toutepuissance hallucinatoire infantile, si bien décrite par la psychanalyse anglo-saxonne. C'est ainsi que, au temps du nécessaire désenchantement pubertaire des dieux infantiles, les adolescents se vouent à la passion mystique du nouveau dieu social : 1'homme, c'est-à-dire eux-mêmes. » ${ }^{12}$ Il n'est donc pas anodin que les thèmes des facultés latentes ou du « potentiel humain » croisent ceux de la latence et du devenir adulte.

\footnotetext{
$9 \quad$ Le Maléfan, 2008, p. 715.

10 Askenazy et al., 2009, p. 30.

11 Douville, 2004, p. 90-93.

12 Lesourd, 2008, p. 19.
} 


\section{Psychiser le maître absolu : solutions pubertaires par le paranormal}

L'intérêt d'une majorité d'adolescents pour le paranormal, comme théorie et comme expérience, se lit dans les statistiques de divers sondages, au point de l'élever au rang d'une banalité. Le contact avec le paranormal peut être motivé par la curiosité et l'ennui (Mischo, 1991), mais aussi impliquer des enjeux plus profonds dans un questionnement de la mort. Ainsi, le recours à une séance de spiritisme peut être une modalité d'exploration des limites du symbolique et des effets du signifiant (Le Maléfan, 2008). Cette mise en scène de la mort peut aussi apparaître, paradoxalement, comme une des formes du fantasme d'immortalité dégagé par Gutton (1993) au temps pubertaire. Ce fantasme peut se développer pour devenir une solution provisoire pour élaborer un deuil ou un traumatisme. Nous essayerons de montrer, au travers de cas cliniques, comment l'adolescence, pensée comme un temps logique proche des états limites, peut subjectiver la mort, ce maître absolu, en la «psychisant ", c'est-à-dire en se rapportant à des pratiques cultivées depuis plus d'un siècle par les sciences psychiques et le spiritisme.

Mots clefs : Croyances à l'adolescence - Fantasme d'immortalité - Pratiques occultes - Expériences paranormales - Hallucinations non psychotiques

\section{Death and psychic experiences - teenage solutions using paranormal}

Research clearly demonstrates that many teenagers have interests in paranormal phenomena (both theoretically, and in terms of experiences and practices), something becoming a commonness. The main self-reported motives of teenagers reflect both curiosity and boredom (Mischo, 1991), but can also implies deeper issues including facing death. Spiritist and occult practices can be a modality of exploration of the limits of the symbolic and of the effects of the signifier (Maléfan, 2008). This production of death can also appear, paradoxically, as one form of the fantasies of immortality described by Gutton (1993) in the juvenile time. This fantasy can develop to become a temporary solution to elaborate a bereavement or a trauma. We would try to show, through clinical cases, how the adolescence, thought as a logical time close to borderline states, can subjectivate death, this "absolute master", by "psychic experiences", i.e. related to practices cultivated for more a century by psychic sciences and spiritualism.

Key words : Beliefs of teenagers - Fantasy of immortality - Occult practices - Paranormal experiences - Non-psychotic hallucinations

EVRARD Renaud, renaud.evrard@etu.univ-rouen.fr, 2 place Galilée, 67380 Lingolsheim. Psychologue, Doctorant à l'Université de RouenEquipe Traumatismes Individuels et Familiaux (TIF) du Laboratoire Psychologie Neurosciences Cognition Affectivité (PSY-NCA). 


\section{$\underline{\text { Références }}$}

Askenazy, F., Dupuis, G., Dor, E., Lestideau, K., Meynadier, A., \& Myquel, A. (2009). Clinique des hallucinations auditives chez l'enfant non-psychotique. Neuropsychiatrie de l'enfance et de l'adolescence, 57, 25-31.

Calvesi, A. (1983). The analytic relationship and its therapeutic factors from a parapsychological viewpoint. Psychoanalytic Review, 70(3), 387-402.

Coulombe, D. (2003). Le fantastique religieux et l'adolescence. Montréal : Fides.

Delaroche, P., \& Mouras, J.-P. (dir., 2004). Peut-on devenir fou ? Paris : Erès, coll. «Le Bachelier ».

Delestre, A. (1997). La religion des étudiants. Paris : L'Harmattan.

Durastante, R. (2009). Du transgénérationnel à la mort désavouée : les jeux d'asphyxie. In: Y. Morhain \& R. Roussillon (dir.), Actualités psychopathologiques de l'adolescence (pp. 143170). Bruxelles : De Boeck Université.

Douville, O. (2004). D'un devenir qui prend le tour de la folie, ou des séductions adolescentes par le franchissement. In : P. Delaroche, \& J.-P. Mouras (dir.), Peut-on devenir fou ? (pp. 71-93). Paris : Erès, coll. « Le Bachelier ».

Evrard, R. (2008). Adolescence et occultisme : mise en perspective différentielle. Mémoire de Master 2 Recherche «Psychopathologie et études psychanalytiques », Faculté de Psychologie et de Sciences de l'éducation, Université Louis-Pasteur, Strasbourg.

Goulding, A. (2005). Healthy schizotypy in a population of paranormal believers and experients. Personality and Individual Differences, 38 , 1069-1083.

Gutton, P. (1993). Essai sur le fantasme d'immortalité à la puberté. Cliniques méditerranéennes, 39/40, 141-154.

Gutton, P. (1996). Adolescens. Paris : PUF.

Hergovich, A., Schott, R., \& Arendasy, M. (2008). On the relationship between paranormal belief and schizotypy among adolescents. Personality and Individual Differences, 45(2), 119-125.

Kardec, A. (1861). Le livre des médiums. Paris : Vermet.

Klosinski, G. (1997). Sectes : Alerte aux parents. Paris : Brepols.

Lacan, J. (1975). Le Séminaire XX: Encore. Paris : Seuil.

Larøi, F., Van der Linden, M., Goëb, J.-L. (2009). Hallucinations et idées délirantes chez les enfants et adolescents : mise en perspective avec les travaux réalisés chez l'adulte. Neuropsychiatrie de l'enfance et de l'adolescence, 57, 32-37.

Le Vallois, Ph., \& Aulenbacher, Ch. (2006). Les ados et leurs croyances. Comprendre leur quête de sens et déceler leur mal-être. Paris : Editions de l'Atelier/Editions ouvrières.

Le Maléfan, P. (2001). Les expériences de mort imminente (EMI) au regard de la psychanalyse. Perspectives psychiatriques, 40(2), 125-132.

Le Maléfan, P. (2005). La « sortie hors du corps » est-elle pensable par nos modèles cliniques et psychopathologiques? Essai de clinique d'une marge. A propos d'un cas. L'Evolution psychiatrique, 70(3), 513-534.

Le Maléfan, P. (2008). «La vérité est ailleurs ». La place du paranormal à l'adolescence comme mode de traitement du réel pubertaire. Adolescence, 26(3), 709-721.

Lesourd, S. (2008). L'incontournable passion mystique de l'adolescent. Adolescence, 26(1), 9-21.

Lucadou, W. v. (1992a). Psyche und Chaos - Jugendliche im Umgang mit dem Okkulten. In: Nr. 16 der Schriftenreihe der Arbeitsgemeinschaft für Gefährdetenhilfe und Jugendschutz in der Erzdiözese Freiburg e.V. (AGJ) (pp. 5-42), Freiburg.

Lucadou, W. v. (1992b). Beten oder Beschwören - Jugendliche auf der Suche nach verlässlichen Antworten. KU-Praxis, 30, 81-94.

Lucadou, W. v., \& Zahradnik, F. (2001). Bericht über die Beratungssituation von Jugendlichen mit ungewöhnlichen Erfahrungen. In: Aktuelle Tipps, Hintergrundmaterialien zum Themenbereich 
sog. Sekten und Psychogruppen (pp. 63-77), September 2001 3/01, Ministerium für Kultus und Sport, Baden-Württemberg.

Maleval, J.-C. (2000). La forclusion du Nom-du-Père. Le concept et sa clinique. Paris : Seuil, «Champ freudien ».

Mischo, J. (1991). Okkultismus bei Jugendlichen: Ergebnisse einer empirischen Untersuchung. Mainz : Matthias-Grünewald-Verlag.

Renard, J.-B. (1988). Les extra-terrestres. Paris : Cerf/Fides, coll. « Bref ».

Roussillon, R., Chabert, C., Ciccone, A., Ferrant, A., Georgieff, N., Roman, P. (2007) Manuel de psychologie et de psychopathologie clinique générale. Paris : Masson.

Schofield, K., \& Claridge, G. (2007). Paranormal experiences and mental health: Schizotypy as an underlying factor. Personality and Individual Differences, 43(7), 1908-1916.

Staehler, F. (2008). Le rapport des adolescents à la religion dans le contexte de l'enseignement religieux catholique en Alsace. Mémoire de Master Recherche, Université Marc Bloch, Faculté de Théologie et Science des Religions, Strasbourg.

Symann, S., \& Hayez, J.-Y. (2008). Hallucinations chez un enfant non-psychotique. Neuropsychiatrie de l'enfance et de l'adolescence, 56, 27-31. 